\title{
Avaliação dos sinais neurocomportamentais de bebês pré-termo internados em Unidade de Terapia Intensiva Neonatal
}

\section{Assessment of the neurobehavioral signs of preterm infants admitted to Neonatal Intensive Care Unit}

\author{
Aline Caren Santos Otoni ${ }^{1}$, Magali Teresinha Quevedo Grave ${ }^{2}$
}

http://dx.doi.org/10.11606/issn.2238-6149.v25i2p151-8

Ottoni ACS, Grave MTQ. Avaliação dos sinais neurocomportamentais de bebês pré-termo internados em Unidade de Terapia Intensiva Neonatal. Rev Ter Ocup Univ São Paulo. 2014 maio/ ago.;25(2):151-8.

RESUMO: Introdução: A prematuridade, o baixo peso ao nascer e a hospitalização precoce e prolongada são fatores de risco para atraso do desenvolvimento infantil. Objetivo: Descrever os sinais neurocomportamentais de aproximação e retraimento apresentados pelos bebês prematuros durante os cuidados diários em uma UTI Neonatal. Procedimentos metodológicos: Pesquisa de campo, exploratória, descritiva, transversal, de caráter quantitativo. Os dados foram coletados através uma ficha de observação dos sinais neurocomportamentais, utilizando a técnica da observação direta e analisados em uma planilha eletrônica de dados do Microsoft Office Excel 2003 for Windows 7. Resultados: Foram avaliados 11 bebês. Os resultados mostram que os bebês apresentaram baixo índice de sinais de aproximação presentes, não emitindo resposta positiva durante as intervenções. Conclusão: Considerando os aspectos próprios da prematuridade e as implicações desta para o desenvolvimento do recém-nascido, é de extrema importância a promoção de práticas que contribuam para o desenvolvimento saudável do recém-nascido.

DESCRITORES: Terapia Ocupacional; Prematuro; UTI Neonatal; Humanização da assistência.
Ottoni ACS, Grave MTQ. Assessment of the neurobehavioral signs of preterm infants admitted to Neonatal Intensive Care Unit. Rev Ter Ocup Univ São Paulo. 2014 maio/ago.;25(2):1518.

ABSTRACT: Introduction: Prematurity, low birth weight and early and prolonged hospitalization are risk factors for developmental delay in children. Objective: To describe the neurobehavioral signs of avoidance cues and approach behavior presented by preterm infants during daily care in a NICU. Methodological procedures: Field research, exploratory, descriptive, cross-sectional quantitative character. Data were collected through a plug observation of neurobehavioral signs, using the technique of direct observation and analyzed in a spreadsheet of data from Microsoft Office Excel 2003 for Windows 7. Results: A total of 11 babies. The results show that the babies had low index signals present approach, not emitting positive response during interventions. Conclusion: Considering the specific aspects of prematurity and its implications for the development of the newborn is of utmost importance promoting practices that contribute to the healthy development of the newborn.

KEYWORDS: Occupational Therapy; Premature; NICU; Humanization of Assistance.

\footnotetext{
*Estudo apresentado ao Centro Universitário Univates/lLajeado/RS como trabalho de conclusão de curso, sendo parte integrante dos requisitos para obtenção do título de especialista em Ações em Estimulação Precoce.

Instituições envolvidas: Fundação de Saúde Pública São Camilo de Esteio, RS e Centro Universitário Univates, Lajeado, RS.

1. Graduada em Terapia Ocupacional pela Universidade Federal de Minas Gerais (UFMG), especialista em Ações em Estimulação Precoce pelo Centro Universitário Univates.

2. Graduada em Fisioterapia pela UFSM, RS, mestre em Desenvolvimento Regional UNISC/RS, doutora em Ciências da Saúde/ Neurociências pela PUCRS. Docente do Centro Universitário Univates, Lajeado, RS.

Endereço para correspondência: Estrada da Bica, n²42 Apt $^{\circ}$ 101, Bairro Cacuia. CEP: 21931-070. Rio de Janeiro, RJ. E-mail: alinecarenotoni@hotmail.com
} 


\section{INTRODUÇÃO}

prematuridade, o baixo peso ao nascer
e a hospitalização precoce e prolongada
ãa fatores de risco para atraso do desenvolvimento infantil. Esses bebês possuem risco de apresentar distúrbios, tanto transitórios quanto permanentes nas várias áreas e componentes do desempenho. A incidência no número de nascimentos de bebês pré-termos continua em crescimento e como conseqüência a permanência na Unidade de Terapia Intensiva Neonatal, onde os recémnascidos são submetidos a diversos tipos de procedimentos, muitas vezes causadores de dor e estresse ${ }^{1}$.

A Organização Mundial de Saúde (OMS) define como recém-nascido pré-termo toda criança nascida antes de 37 semanas de gestação. O número de nascimentos de bebês pré-termo cresce constantemente, observandose assim o aumento da sobrevivência de bebês de alto risco e consequentemente o índice de crianças com sequelas graves, influenciando na qualidade de vida dessa população ${ }^{2,3}$. Atualmente cerca de 7 a $15 \%$ do total dos nascimentos vivos representam a incidência de nascimentos de recém-nascidos prematuros com baixo peso, sendo de 20 milhões o número de nascimentos de bebês prematuros no mundo ${ }^{3}$.

As causas da prematuridade estão relacionadas aos fatores ambientais desfavoráveis no período pré-natal. Estes fatores atuam sobre a condição de saúde da mãe e do bebê e podem acarretar no nascimento prematuro. Os principais fatores que predispõem ao nascimento prematuro são: idade materna; nutrição materna; drogas e radiação; doenças e distúrbios maternos; fator Rh incompatível; estresse materno ${ }^{4}$.

Os avanços na área da Neonatologia têm favorecido de forma significativa para aumentar o número de sobrevivência de bebês nascidos pré-termo ${ }^{5}$. O bebê que nasce pré-termo ainda teria algumas semanas de vida intra uterina para desenvolver estruturas anatômicas e capacidades que the assegurariam qualidade de vida fora do ventre materno. O recém-nascido pré-termo (RNPT), por necessitar de continuação da sua gestação fora do útero materno, encontra-se em uma situação de fragilidade, pois seu Sistema Nervoso Central não possui as condições básicas para enfrentar e organizar os estímulos advindos de um ambiente desconhecido e desfavorável, porém necessário para a sua sobrevivência ${ }^{1,6}$. Existem pesquisas que apontam que os riscos para alterações no desenvolvimento são maiores, quanto menor o peso ao nascimento do bebê. Tal fato é explicado pela ampla diversidade de complicações clínicas que o recém-nascido pode apresentar durante o período de internação na UTI Neonatal por questões do próprio ambiente da UTI como iluminação excessiva e ruído elevado, e pelas rotinas da equipe como manuseio freqüente, procedimentos dolorosos e o desrespeito dos períodos de repouso. Dessa forma, a UTI Neonatal se torna uma ambiente estressante para o bebê, evidenciado que os estímulos que o pré-termo deveria receber para favorecer o seu desenvolvimento são inadequados ${ }^{1}$.

Durante as intervenções, as respostas comportamentais dos bebês prematuros a diversas situações que geram desconforto ou prazer, podem se mostrar a princípio inespecíficas. Muitos profissionais podem assim não observarem ou reconhecerem esses sinais. No entanto, é preciso que tais sinais sejam identificados, a fim de que os bebês tenham suas individualidades respeitadas e recebam uma intervenção que promova um desenvolvimento saudável ${ }^{2}$.

No ventre materno o feto passa por constantes transformações. Recebe estímulos do ritmo circadiano materno, estímulo tátil através do contato com as paredes uterinas, que também são responsáveis pelo controle da força da gravidade, estímulos vestibulares provenientes não só do movimento do corpo da mãe, como também das suas próprias trocas de posições e estímulos proprioceptivos advindos dos seus movimentos e posicionamentos corporais. Além disso, dorme a maior parte do tempo da sua vida intrauterina, permitindo assim que seu organismo em desenvolvimento adquira gradualmente capacidade para incorporar os diversos estímulos, de forma adequada e controlada, a partir do seu nascimento ${ }^{1,6}$.

A literatura aponta que o fato do bebê prétermo encontrar-se fora do seu ambiente natural, o ventre materno, justamente em um período crítico para o seu desenvolvimento, resulta em alteração no seu desenvolvimento neurológico e psicomotor, sendo mais prejudicial quando menor a idade gestacional da criança ${ }^{6}$. Ao nascer prematuramente, o bebê geralmente necessita de internação na Unidade de Terapia Intensiva Neonatal para receber os cuidados necessários. Ao mesmo tempo em que é um ambiente que salva, também pode ser o lugar que deixará marcas negativas, como por exemplo, uma lesão no encéfalo. Lesão que pode ocorrer por um desconhecimento dos profissionais que lidam com esse bebê e não pela má vontade dos mesmos ${ }^{1}$.

Os procedimentos médicos e da enfermagem podem requerer frequentes manipulações do recém-nascido, implicando em estimulação tátil adversa, com eventual geração de dor. Ao serem submetidos a tais procedimentos, os bebês pré-termos gasta energia para suportar a dor, energia essa que seria utilizada para garantir a vida e o 
desenvolvimento. Além disso, o bebê permanece em um ambiente com luz e ruídos constantes, o que somado ao fato citado anteriormente, se traduz na perda ou alteração do estabelecimento do ritmo circadiano ${ }^{1,7}$.

A pesquisadora Heidelise Als e seus colaboradores iniciaram, em 1978, pesquisas sobre o comportamento e assistência ao recém-nascido de baixo peso, tendo como premissa uma prática distinta de cuidados para o desenvolvimento, cujos resultados possibilitaram a elaboração de uma prática individualizada para favorecer o desenvolvimento dos bebês prematuros, proporcionando ganhos para o desenvolvimento concreto das diversas funções do organismo, especialmente das áreas cognitiva, motora e comportamental ${ }^{4}$. A Teoria Síncrono-ativa do Desenvolvimento (TSAD) proposta pela pesquisadora enfatiza o acolhimento humanizado aos recém-nascidos e recomenda os passos a serem seguidos para a percepção do funcionamento cerebral do bebê, através do seu comportamento. A teoria discute o limite que o bebê possui em relação ao estresse e sua capacidade de auto-regulação em relação a ele, emitindo respostas sobre a sua disponibilidade para as intervenções de acordo com o funcionamento de cada subsistema (autônomo, motor, estados comportamentais, atenção e interação social e sistema regulador ${ }^{2,8}$.

Todos esses subsistemas são interligados e interagem entre si, provendo o estado de homeostase do bebê, sendo esta a função primordial do bebê pré-termo, mesmo recebendo constantes estímulos em seus subsistemas que não se encontram preparados para tal. Portanto, esses estímulos podem favorecer ou prejudicar a estrutura cerebral $^{8}$. Caso ocorra uma desorganização em algum dos subsistemas, uma sobrecarga acontecerá nos demais e será manifestado pelo RNPT através da mudança de comportamento, apresentando dificuldade na interação ${ }^{2,8}$.

Dessa forma, a TSAD nomeia como sinais de aproximação ou retraimento as alterações comportamentais dos bebês pré-termos em resposta aos estímulos advindos do ambiente. Os sinais de aproximação indicam que o recém-nascido está recebendo a quantidade de estimulação e interação adequada, sendo este o momento do profissional intervir com ele. Já os sinais de retraimento demonstram que o bebê está muito estressado e as estimulações excessivas devem ser gradativamente retiradas, mostrando ao profissional que esse não é o momento propício para realizar intervenção, sendo necessário aguardar mais um pouco².

Sabendo da importância em reconhecer os sinais de aproximação ou retraimento para prevenir sequelas e garantir qualidade de vida, o presente estudo objetivou descrever a frequência dos sinais neurocomportamentais de aproximação e retraimento apresentados pelos bebês prematuros durante os cuidados diários em uma UTI Neonatal.

\section{PROCEDIMENTOS METODOLÓGICOS}

O presente estudo se classifica como uma pesquisa de campo, exploratória, descritiva, transversal, de caráter quantitativo $^{9}$ e foi realizado na Unidade de Terapia Intensiva Neonatal de uma Fundação de Saúde Pública de um município da grande Porto Alegre - RS, no período de 16/11/2013 a 31/03/2013. Esta UTIN possui 06 leitos destinados aos recém-nascidos de até 28 dias.

A amostra foi constituída por 11 bebês cujos pais ou responsáveis puderam ser contatados pela pesquisadora ao término do horário de visitas e convidados a se dirigirem a sala de espera, localizada no corredor da UTI, no intuito de que os objetivos e metodologia da pesquisa lhes fossem apresentadas. Foram incluídos na pesquisa, bebês prétermos que estiveram internados na UTI neonatal no período de novembro de 2012 a março de 2013, em condições estáveis, aptos a serem observados, mediante liberação prévia do médico pediatra responsável pela UTI neonatal da referida instituição e cujos pais ou responsáveis assinaram o Termo de Consentimento Livre e Esclarecido (TCLE). Os critérios de exclusão se aplicaram a recém-nascidos internados na UTI neonatal com idade gestacional superior a trinta e sete semanas; bebês que possuíssem patologias ou síndromes incompatíveis com a vida (anencefalia, síndrome de Edward, dentre outras); bebês prematuros que, por qualquer situação de emergência, necessitaram, por exemplo, de uso de ventilação mecânica, monitorização hemodinâmica.

Utilizou-se como instrumento de coleta de dados uma ficha de observação dos sinais neurocomportamentais, elaborada pela pesquisadora sob a perspectiva da Teoria Síncrono-Ativa de Desenvolvimento (TSAD), que contém itens dos comportamentos de aproximação e retraimento, a serem assinalados se presente ou ausentes. Segundo a TSAD, os recém-nascidos emitem respostas aos estímulos advindos do meio através de alterações comportamentais observáveis, sendo nomeadas como sinais de aproximação ou retraimento. Estes sinais indicam se o bebê está recebendo uma quantidade de estimulação e interação adequada acenando para o profissional que esse é o momento de intervir com ele ou se o bebê está muito estressado e as estimulações excessivas devem ser retiradas gradualmente, indicando ao profissional a necessidade de aguardar um momento mais adequado para realizar a intervenção, respectivamente . $^{8}$

Foram descritos ainda na ficha de observação 
dados relativos quanto à presença de ruídos e luminosidade do ambiente. Informações relativas aos fatores que levaram ao parto prematuro, a idade gestacional, peso ao nascimento e índice APGAR foram coletadas em prontuário a partir do registro médico.

A coleta de dados ocorreu no período de $16 / 11 / 2013$ a $31 / 03 / 2013$. Esta etapa foi desenvolvida durante a troca de fraldas, elegendo-se o horário das 17 horas. A troca de fralda é realizada em horários prédeterminados, sendo a escolha do horário das 17 horas por ser um momento que causaria menor interferência na rotina da equipe. A técnica aplicada foi a de observação direta dos bebês antes, durante e após o procedimento, sendo que foi feito o preenchimento da ficha de observação dos sinais concomitantemente. Os dados relativos à frequência cardíaca, frequência respiratória e saturação de oxigênio foram colhidos do painel das incubadoras e folha de sinais. Os dados quantitativos foram analisados e armazenados em uma planilha eletrônica de dados do Microsoft Office Excel 2007 for Windows 7, onde receberam tratamentos estatísticos descritivo, sendo apresentados na forma de gráficos.

Durante o procedimento da troca de fraldas desempenhado pela equipe de enfermagem, foi utilizado o recurso da filmagem com câmera digital para análise precisa dos comportamentos apresentados pelos bebês. A pesquisadora permaneceu à beira do leito realizando a filmagem para registro do comportamento dos bebês, enquanto a equipe de enfermagem realizava a troca de fraldas. Os dados coletados na filmagem foram repassados para a ficha de observação e submetidos a análise.

A pesquisa foi submetida e aprovada, em 15 de novembro de 2012, pelo Comitê de Ética e Pesquisa do Centro Universitário Univates, parecer número 147.169, respeitando a Resolução 196/96, referente à pesquisa com seres humanos. Além disso, necessitou também de assinatura de consentimento da direção da fundação onde foi desenvolvida. Os dados foram coletados depois de aceite dos pais e/ou responsáveis dos bebês pela assinatura do Termo de Consentimento Livre e Esclarecido.

\section{RESULTADOS}

A população da pesquisa foi constituída por 11 bebês pré-termo, sendo $07(63,6 \%)$ do sexo masculino e $04(36,3 \%)$ do sexo feminino. A idade gestacional variou entre 28 e 36 semanas (média de 33,09 semanas). O peso ao nascer permaneceu entre 1084 g e 2805 g (média de 1952,72 g). Os dados sobre as características gerais dos RN estão apresentados na Tabela 1 .

Tabela 1 - Características gerais dos bebês pré-termo, participantes do estudo, internados em uma Unidade de Terapia Intensiva Neonatal

\begin{tabular}{|c|c|c|c|}
\hline \multicolumn{2}{|c|}{ Características gerais } & População (N=11) & Média / Porcentagem (\%) \\
\hline \multicolumn{2}{|c|}{ Peso ao nascer } & $1084 \mathrm{~g}-2805 \mathrm{~g}$ & 1952,72 \\
\hline \multicolumn{2}{|c|}{ Idade gestacional } & 28 - 36 semanas & 33,09 semanas \\
\hline \multicolumn{4}{|l|}{ Sexo } \\
\hline & Feminino & 04 & $36,30 \%$ \\
\hline & Masculino & 07 & $63,60 \%$ \\
\hline \multirow[t]{2}{*}{ Apgar } & 1 , & 3 a 9 & \\
\hline & 5 & 6 a 10 & \\
\hline
\end{tabular}

Os fatores que levaram ao parto prematuro, conforme registro médico em prontuário, foram bolsa rota e infecção do trato urinário. Ressalta-se ainda que em alguns prontuários não constava o registro sobre a causa da prematuridade. Verificou-se junto ao Serviço de Arquivo Médico e Estatística (SAME) que ocorreram 23 nascimentos de bebês prematuros no período entre novembro de 2012 a março de 2013, sendo que 21 RNPT necessitaram de internação na UTI Neonatal. Foram registrados 02 óbitos nesse mesmo período.

Em relação ao tempo de permanência de internação na UTI neonatal dos bebês participantes do estudo, houve uma média de 27,9 dias. Foi observado que $37 \%$ dos RNPT permaneceram por um período acima de 30 dias. $27 \%$ ficaram internados por um período inferior a 10 dias, outros $9 \%$ permaneceram por um período de até 20 dias, e os demais $27 \%$ por um período de até 30 dias (Gráfico 1). 
Gráfico 1 - Permanência na UTI Neonatal dos bebês pré-termo participantes do estudo

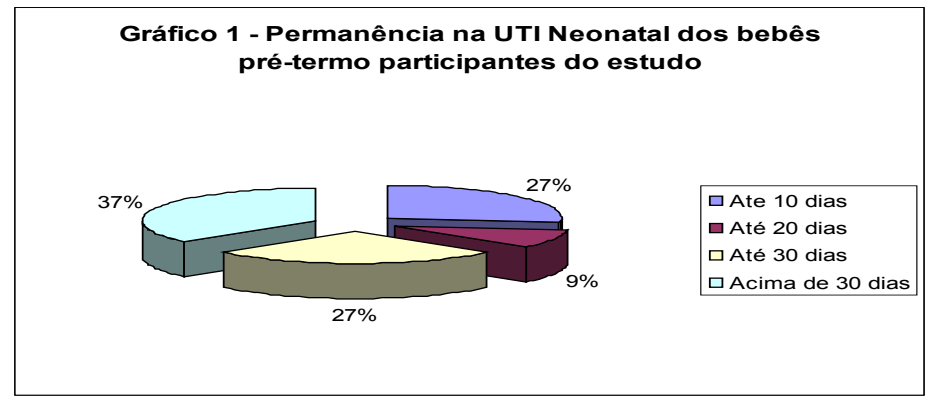

Foram observados os sinais de aproximação e retraimento dos bebês referente ao funcionamento dos subsistemas autonômico, motor, estados comportamentais e atenção e interação social. A quantidade de comportamentos observados para cada subsistema consta na Tabela 2.

Tabela 2 - Quantificação de itens observados nos subsistemas autonômico, motor, estados comportamentais e atenção e interação social

\begin{tabular}{lcc}
\hline Subsistemas & $\begin{array}{c}\text { Número de comportamentos } \\
\text { de aproximação avaliado }\end{array}$ & $\begin{array}{c}\text { Número de comportamentos de } \\
\text { retraimento avaliado }\end{array}$ \\
\hline Autonômico & 5 & 8 \\
Motor & 8 & 5 \\
Estados comportamentais & 1 & 4 \\
Atenção e interação social & 1 & 1 \\
Total & 15 & 18 \\
\hline
\end{tabular}

$\mathrm{Na}$ Tabela 3 verificam-se as respostas individuais para os sinais de aproximação e retraimento de cada bebê observado. Do total de 11 recém-nascidos, apenas 04 apresentaram maior número de sinais de aproximação presentes, mas também valor pouco significativo. $\mathrm{O}$ bebê da amostra de número 10 chama a atenção pelo fato de possuir maior número de sinais de retraimento presentes que ausentes.

Tabela 3 - Quantificação das respostas encontradas em cada bebê pé-termo participante do estudo

\begin{tabular}{ccccc}
\hline & \multicolumn{2}{c}{ Sinais de aproximação } & \multicolumn{2}{c}{ Sinais de retraimento } \\
\hline $\mathbf{N}^{\mathbf{0}}$ da amostra & Presente & Ausente & Presente & Ausente \\
\hline 01 & 8 & 7 & 5 & 13 \\
02 & 9 & 6 & 4 & 14 \\
03 & 6 & 9 & 3 & 15 \\
9 & 9 & 6 & 2 & 16 \\
05 & 7 & 8 & 6 & 12 \\
06 & 7 & 8 & 4 & 14 \\
07 & 7 & 8 & 5 & 13 \\
08 & 7 & 8 & 6 & 12 \\
09 & 8 & 7 & 4 & 14 \\
10 & 4 & 11 & 7 & 11 \\
11 & 5 & 10 & 4 & 14 \\
TOTAL & 77 & 88 & 50 & 148 \\
\hline
\end{tabular}


Os resultados mostram que os bebês apresentaram 46,67\% dos sinais de aproximação presentes e $53,33 \%$ dos sinais de aproximação ausentes durante o procedimento da troca de fraldas. Já em relação aos sinais de retraimento, $25,25 \%$ foram presente e
$74,75 \%$ estiveram ausentes (Gráfico 2). Analisando estes resultados, observa-se que os bebês não emitiam resposta positiva durante a intervenção. Em contrapartida, não foram observados sinais significativos de estresse no procedimento.

Gráfico 2 - Respostas encontradas nos bebês pré-termo participantes do estudo durante troca de fraldas na Unidade de Terapia Intensiva Neonatal

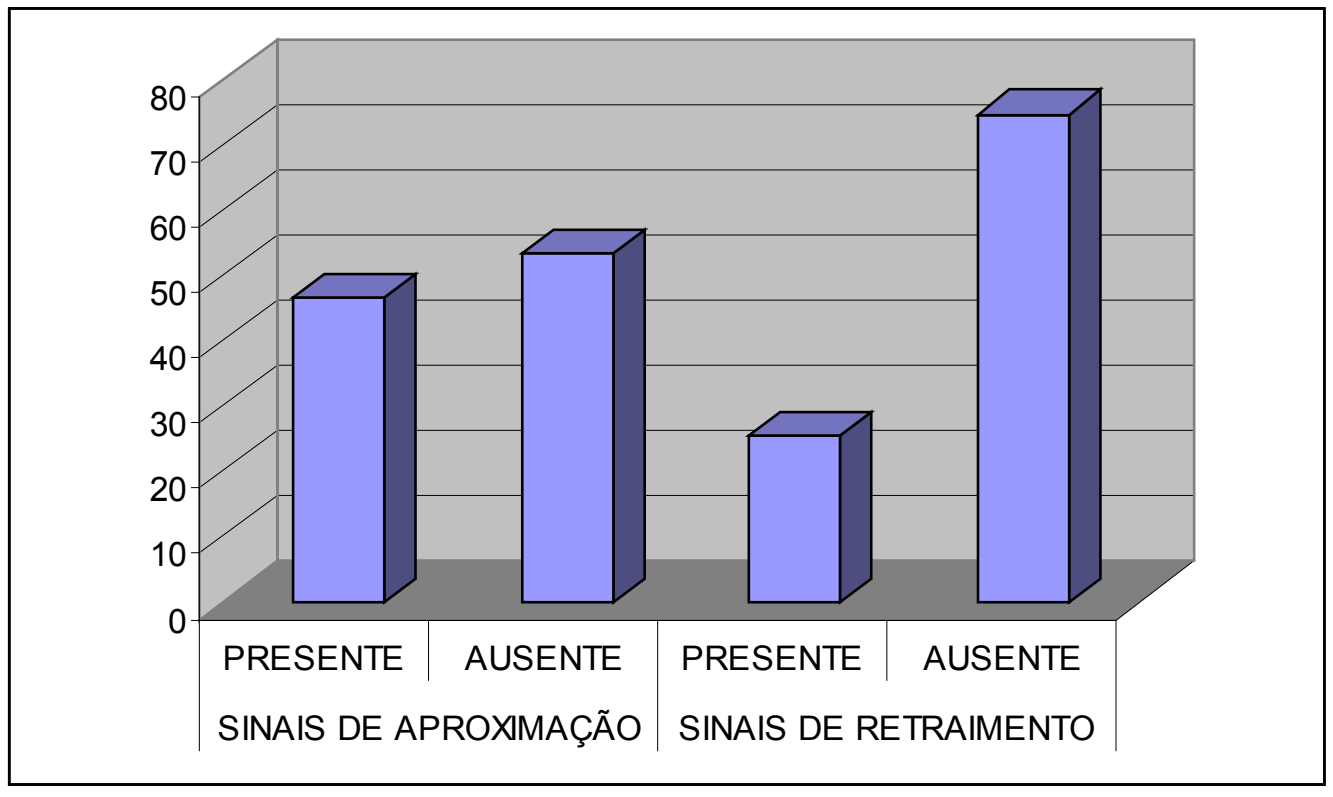

Quanto ao ambiente da unidade de terapia intensiva neonatal, observaram-se algumas condições desfavoráveis, tais como: ruído elevado como toque de telefone, ar condicionado ligado e iluminação excessiva, visto que a luz permanece constantemente acesa, não sendo controlada sua intensidade.

\section{DISCUSSÃO}

A Política Nacional de Humanização (PNH) tem como um dos seus princípios norteadores a observação e escuta atenta, sendo capaz de perceber as diversas demandas do usuário ${ }^{10}$. Sendo assim, a prática de observação dos sinais neurocomportamentais de cada bebê é uma ação que contempla a nova sistemática de humanização proposta pelo Sistema Única de Saúde (SUS).

O bebê pré-termo possui como função primordial manter o equilíbrio do seu organismo, sendo que a Teoria Síncrono-Ativa oferece subsídios para que os profissionais que atuam com essa população, ofereçam uma abordagem humanizada, evitando o surgimento de sequelas. Por sua vez, o ambiente da UTI necessário para a sobrevivência do RNPT também requer atenção e a adoção de medidas como controle da iluminação, ruído, temperatura e manipulações podem auxiliar na recuperação e desenvolvimento saudável do bebê pré-termo.

Farias $^{4}$ analisou os comportamentos de aproximação e retraimento de 19 bebês internados em uma UTI Neonatal evidenciando uma prevalência dos sinais de retraimento sobre os de aproximação, demonstrando que os bebês estavam desorganizados em termos fisiológicos. A diferença dos achados da presente pesquisa, em que os sinais de retraimento não se mostraram significativos, podem se justificar pelo tempo de observação, tendo em vista que os bebês foram observados apenas em único momento. Mas desperta atenção o fato de que os sinais de aproximação não foram identificados presentes em sua maioria, reforçando assim a necessidade de avaliar os sinais neurocomportamentais de aproximação e retraimento durante os cuidados diários na UTI Neonatal. 
Almohalha e Guerra ${ }^{2}$ procuraram investigar o nível de identificação dos sinais neurocomportamentais por parte dos profissionais de uma UTIN e constataram que os profissionais de nível superior conseguem reconhecer mais sinais que os de nível técnico. Como possíveis fatores que influenciam para correta identificação desses sinais encontraram extensiva jornada de trabalho, sutileza dos sinais apresentados pelos bebês, experiência profissional e a falta de capacitação.

Em pesquisa realizada por Cardoso et al. ${ }^{1}$ (2010) com 14 bebês pré-termos, de ambos os sexos, com peso de nascimento inferior a $1.500 \mathrm{~g}$ internados em UTIN verificou-se a existência de dor nos de cuidados diários avaliados (pesagem, troca de fraldas e posicionamento). Também em relação ao ambiente da UTI Neonatal foram encontradas condições desfavoráveis tais como ruído elevado, iluminação excessiva e manuseio frequente. De Sousa et al. ${ }^{11}$ constataram alto índice de manipulações de RNPT em UTIN, não havendo tempo para descanso, em pesquisa realizada com 09 bebês prematuros na UTIN de um hospital da rede pública no município de São Paulo.

Em ambos os estudos foram apontados o fato de que apesar das ações de cuidados serem necessárias para a qualidade de vida do bebê, as mesmas são feitas seguindo a rotina estabelecida pela UTI Neonatal e não às condições fisiológicas do recém-nascido. Essa situação também foi evidenciada na presente pesquisa, corroborando a necessidade de se pensar outras formas de realização desses procedimentos, tornando-os menos prejudiciais ao desenvolvimento dos RNPTs.

Em um levantamento bibliográfico realizado por Resende e Fernandes ${ }^{12}$, os autores também encontraram a exposição do recém-nascido a situações que geram dor e estresse, o que pode acarretar complicações que dificultam a manutenção da homeostase e aumentam as chances de óbito. Com a diminuição da mortalidade neonatal, há aumento no número de morbidades nessa população, sendo um fator determinante para o prognóstico da mesma, a qualidade do atendimento prestado durante a hospitalização ${ }^{13}$. Além disso, esses bebês não são encaminhados para acompanhamento após o egresso da UTIN, fato também averiguado por Méio et al ${ }^{13} \mathrm{em}$ estudo realizado no Estado do Rio de Janeiro.

Diante dos dados encontrados na literatura e os resultados encontrados neste estudo, observa-se a necessidade de sensibilização dos profissionais da equipe da UTI Neonatal, quanto à importância da observação dos sinais neurocomportamentais a fim de que se promovam práticas que contribuam para o desenvolvimento saudável do recém-nascido. Tais observações pertencem às ações terapêuticas ocupacionais em UTI Neonatal que podem ser desenvolvidas com intervenções e orientações no ambiente físico, com a equipe de enfermagem e com o recém-nascido e sua família ${ }^{14}$.

O estudo aponta para a importância dos profissionais que atuam em UTIN conhecerem o problema da sobrecarga de estímulos adversos que geram desequilíbrio no bebê e desenvolverem estratégias para reduzir os sinais de estresse, de modo a contribuir para que o bebê possa adquirir as habilidades necessárias para garantir um desempenho ocupacional exitoso a médio e longo prazo $^{6}$.

Avilès ${ }^{6}$ pontua que a intervenção da Terapia Ocupacional no ambiente da UTIN justifica-se por esta profissão compreender a necessidade de proteger o bebê e favorecer seu desenvolvimento neuropsicomotor, entendendo que nesta primeira fase de vida serão estruturados aspectos para o desenvolvimento global e desempenho funcional. Os terapeutas ocupacionais na atenção desta população atuam na aplicação de intervenções destinada a adequar os estímulos do ambiente ao mesmo tempo em que apóiam os esforços de autoregulação dos bebês, com o objetivo de favorecer um adequado desenvolvimento.

\section{CONCLUSÕES}

Considerando os aspectos singulares intrínsecos e próprios da prematuridade e as implicações desta para o desenvolvimento do recém-nascido, é de extrema importância a promoção de práticas que contribuam para o desenvolvimento saudável do recém-nascido. Além disso, torna-se necessário a sensibilização dos profissionais da equipe que atuam com recém-nascidos prematuros quanto à importância de observar os sinais faciais e corporais dos bebês que podem ser indícios de dor ou estresse, proporcionando uma melhor qualidade no atendimento prestado e consequentemente aumento na qualidade de vida desta população.

Um melhor desenvolvimento global do recémnascido poderá ser alcançado a partir da modulação correta dos estímulos, sendo que para isso, os profissionais devem estar aptos para compreender e analisar tais sinais.

Ressalta-se ainda que esta pesquisa não teve a intenção de questionar ou criticar as medidas adotadas pela UTIN, apenas contribuir para a adoção de práticas que favoreçam prestação de assistência humanizada e apresentar as possibilidades de atuação da Terapia Ocupacional neste contexto. 


\section{REFERÊNCIAS}

1. Cardoso AS, Lima AM, Maximino VS, Specian CM. Estudo exploratório de dor em recém-nascidos pré-termos em uma unidade de tratamento intensivo neonatal. Cad Ter Ocup da Univ Federal São Carlos. 2010;18(2):105-14 [citado 14 abr. 2012]. Disponível em: http://www.cadernosdeterapiaocupacional. ufscar.br/index.php/cadernos/article/viewFile/349/278.

2. Almohalha L, Guerra RMR. Identificação dos sinais neurocomportamentais de bebês pré-termo por profissionais que atuam na Unidade de Terapia Intensiva Neonatal (UTIN). Rev Ter Ocup. Univ São Paulo. 2011;22(2):117-26 [citado 14 abr. 2012]. Disponível em: http://www.revistasusp.sibi.usp. br/pdf/rto/v22n2/05.pdf. DOI: http://dx.doi.org/10.11606/ issn.2238-6149.v22i2p117-126.

3. Medeiros JSS, Mascarenhas MFPT. Banho humanizado em recém-nascidos prematuros de baixo peso em uma enfermaria canguru. Rev Ter Ocup Univ São Paulo 2010;21(1):51-60 [citado 14 abr. 2012]. Disponível em: http://www.revistasusp. sibi.usp.br/scielo.php?script $=$ sci_arttext\&pid $=\mathrm{S} 1415$ 91042010000100008\&lng=pt\&nrm=iso. DOI: http://dx.doi. org/10.11606/issn.2238-6149.v21i1p51-60

4. Farias GRB. Análise dos Comportamentos de Aproximação e Retraimento de Pré-Termos de Risco Evidenciados em uma Unidade de Terapia Intensiva Neonatal [Dissertação]. Belém: Universidade Federal do Pará, Centro de Filosofia e Ciências Humanas, Programa de Pós-Graduação em Teoria e Pesquisa do Comportamento; 2006 [citado 28 abr. 2012]. Disponível em: http://www.ufpa.br/ppgtpc/dmdocuments/MESTRADO/ Dissertacao_Gabriela_Ribeiro.pdf.

5. Linhares MBM, Carvalho AEV, Bordin MBM, Chimello JT, Martinez FE, Uorge SM. Prematuridade e muito baixo peso como fatores de risco ao desenvolvimento da criança. Paidéia, FFCLRP-USP, Ribeirão Preto. 2000;10(18):60-9 [citado 24 abr. 2012]. Disponível em: http://www.scielo.br/ pdf/paideia/v10n18/06.pdf. http://dx.doi.org/10.1590/S0103863X2000000100006

6. Avilès C. Terapia Ocupacional en neonatología: una experiencia desde el sur. Rev Chilena Ter Ocup. 2010;10:9-19. Disponível em: $<$ http://www.revistas.uchile.cl/index.php/RTO/ article/viewFile/10556/10634>. Acesso em: 24 abr. 2012. DOI: 10.5354/0717-6767.2010.10556

7. Cardoso AS, Lima AM, Maximino VS, Specian CM. A observação de bebês na UTI neonatal para verificação de sinais de dor/estresse durante procedimentos rotineiros. 2006 [citado 20 abr. 2012]. Disponível em: http://www.inicepg. univap.br/cd/INIC_2006/inic/inic/03/INIC0000473.ok.pdf.

8. Brasil. Ministério da Saúde. Secretaria de Atenção à Saúde. Departamento de Ações Programáticas Estratégicas. Atenção humanizada ao recém-nascido de baixo peso: método Canguru. 2a ed. Brasília: Editora do Ministério da Saúde; 2011. (Série A. Normas e Manuais Técnicos).

9. Fernandes LA, Gomes JMM. Relatórios de pesquisa nas ciências sociais: características e modalidades de investigação. ConTexto, Porto Alegre. 2003;3(4) [citado 28 maio 2012]. Disponível em: http://seer.ufrgs.br/index.php/ConTexto/ article/viewFile/11638/6840.

10. Alves CA, Deslandes SF, F, Mitre RMA. Desafios da humanização no contexto do cuidado da enfermagem pediátrica de média e alta complexidade. Interface, Botucatu. 2009;13(Suppl.1):581-94 [citado 17 abr. 2013]. Disponível em: http://www.scielo.br/pdf/icse/v13s1/a10v13s1.pdf. http:// dx.doi.org/10.1590/S1414-32832009000500010

11. De Sousa MWCR, Da Silva WC, Neves SA. Quantificação das manipulações em recém-nascidos pré-termo em Unidade de Terapia Intensiva: uma proposta de elaboração de protocolo. ConScientiae Saúde. 2008;7(2):269-74 [citado 27 maio 2013]. Disponível em: http://www.uninove.br/pdfs/publicacoes/ conscientiae_saude/csaude_v7n2/cnsv7n2_3o.pdf.

12. Resende MA, Fernandes JM. Intervenção mínima: um cuidado essencial ao recém-nascido pré-termo em unidade de terapia intensiva neonatal. 2010 [citado 25 maio 2013]. Disponível em: http://www.iptan.edu.br/publicacoes/anuario_prodcientifica/ arquivos/revista1/resumos_expandidos/Resumo_expandido_ Marcio_Juliana.pdf.

13. Méio MDBB, Magluta C, Mello RR, Moreira MEL. Análise situacional do atendimento ambulatorial prestado a recém-nascidos egressos das unidades de terapia intensiva neonatais no Estado do Rio de Janeiro. Cienc Saúde Coletiva. 2005;10(2):299-307 [citado 27 maio 2013]. Disponível em: http://www.scielo.br/pdf/csc/v10n2/a07v10n2.pdf. http:// dx.doi.org/10.1590/S1413-81232005000200007

14. Henrique LR, Cruz OMC, Farias AGF, Rodrigues RB, Guarany NR R. Atuação do terapeuta ocupacional no método mãe canguru. CIC 2011 [citado 23 maio 2013]. Disponível em: http://www.ufpel.edu.br/cic/2011/anais/pdf/CS/CS_00391.pdf. 